\title{
Differences between conventional and psychiatric hospitalization - Suicidality in severe personality disorders
}

\author{
Cesar Garza-Guerrero* \\ Department of Psychiatry, Facultad de Medicina y Hospital Universitario "Dr. José Eleuterio Gonzalez," Autonomous University of Nuevo Leon, \\ Monterrey, Nuevo Leon, Mexico
}

\begin{abstract}
In spite of the enormous advances in differentiating hospitalizations in conventional medical care systems from psychiatric hospitalization, the bidirectional translocation of these prevails, i.e., admission of patients with primary psychiatric disorders to general medicine wards or the application of a traditional medical model to a supposed program of psychiatric hospitalization. In this paper, the divergence of medical hospitalization and psychiatric hospitalization is explored along two dimensions: (1) indications and justification for a psychiatric hospitalization; and (2) suicidality in severe personality disorders. Contrary to the erroneous prediction of few decades ago about the disappearance of the psychiatric hospital, the need for modern psychiatric institutions and psychiatry divisions that integrate the advances and contemporary proposals in the area of mental health and behavioral sciences is more imperative than ever. For this, a continuous effort is essential to critically explore the current situation and address the enormous challenges represented by the training of personnel, professors, and psychiatry residents for the optimal use of available hospital resources.
\end{abstract}

Key words: Psychiatric hospitalization. Borderline personality organization. Characterological suicidality.

\section{Introduction}

A study of the differences between conventional hospitalization and psychiatric hospitalization would seem unnecessary if it was not for the frequent errors caused by not delimiting the modalities of a traditional medical model, from those of a modern psychiatric program. Despite advances in contemporary medical and psychiatric care, bidirectional translocation prevails - the admission of psychiatric patients to conventional medicine wards or the application of a traditional medical model to a supposed psychiatric hospitalization, either in a psychiatry division in a general hospital or an independent psychiatric hospital or clinic. Herein, the differences between medical and psychiatric hospitalization are explored along two dimensions: (1) indications and justification for psychiatric hospitalization and (2) suicidality in severe personality disorders.

\section{Indications and justification for hospitalization}

The reasons for admission to a general hospital are relatively clear: the evaluation, care, treatment, and prevention of bodily dysfunctions, disorders, or diseases. The reasons and justifications for psychiatric hospitalization, on the other hand, entail potential iatrogenic risks that could have been under the control of patients and psychiatrists and staff, but due to ignorance or to a psychopathic or perverse plan, particularly in relation to borderline personality organization (BPO), these were deliberately ignored ${ }^{1-3}$.

\footnotetext{
Correspondence:

*César Garza-Guerrero

E-mail: cesargg@me.com

Date of reception: 17-05-2019

Date of acceptance: 20-08-2019

DOI: 10.24875/RMU.19000092

1665-5796/@ 2019 Universidad Autónoma de Nuevo

(http://creativecommons.org/licenses/by-nc-nd/4.0/).
} 


\section{Risks in the hospitalization of patients with severe personality disorders}

The reluctance to hospitalize patients with a borderline personality disorder (BPO) persists, arguing that extended inpatient care leads to deterioration and adverse events. Nevertheless, results from a longitudinal study of extended inpatient treatment indicate that this can result in a significant and clinically meaningful improvement in patients with $\mathrm{BPO}^{4}$. It should be underscored, however, that this research took place in a clinical setting with a high volume of direct and active, group and individual, therapeutic encounters. An extended hospital setting with scant resources and few groups and individual interventions may create iatrogenic effects. Considering that most conventional inpatient psychiatric units do not have trained personnel or sufficient human resources to care adequately for patients with severe character pathology, it might be worthwhile to keep in mind, the significant risks reported in literature $e^{1,5-7}$. Hospitalization could initiate a regressive deterioration in patients. These could be manifested by the expectation of being passively cared for by an unconditionally benevolent and omnipotent staff (idealization of the projected object relationship) or else, the anticipation of being cruelly subdued and controlled (the aggressively paranoidizing side of the intrapsychic conflict). Furthermore, the validation of a negative identity: "if we have already touched bottom, what does it matter if we just go wild and act crazy," and renouncing any ambition of consolidating a sense of autonomy and a higher level of quality in their lives.

Admission to the hospital could represent a disruption of supportive networks: the abortion of the study projects or the discontinuation of jobs and community commitments. Unfortunately, avoiding these responsibilities is precisely what usually encourages patients with a BPO to force a hospitalization, which in the end only feeds back the secondary gain of their dependence and psychological parasitism ${ }^{5,6,8}$. Patients with a BPO without a consolidated, autonomous, and independent value system coupled with an identity diffusion syndrome are extraordinarily vulnerable to various forms of hospital infection, for example, participation in collective regressive phenomena, identification with new modes of self-destruction, imitation of anorexic behaviors, and pathological collusion with psychopathic deviations, among others ${ }^{1}$. Furthermore, there is the risk of polypharmacy and its negative impact, especially in psychiatric hospital programs without staff trained in personality disorders and psychodynamic procedures; staff forced to reduce their intervention to custody, overmedication, and even patient sedation ${ }^{1,2,5,9,10}$. The problem of overmedication resides in the fact that it restricts hospitalization to a "neurovegetative life" and also impedes the careful planning of ways to return to study, work, or previous commitments 5,6 .

In the decision to hospitalize patients with a BPO, the risks and potential benefits should be explicitly explored $^{11,12}$. Any possibility of hospitalization must start with warning patients with a BPO, and their partners and relatives, that the ideal treatment of their characterological disorder should be on an outpatient basis. The common denominator for admission of patients with a BPO is related to the complication that should have been under the control of the patient, but they were not contained, and they now prevent the beginning or continuation of outpatient treatment. Therefore, the main objective for hospitalization is to understand the nature of these constraints and their prompt control and prevention. From a psychoeducational perspective, it is imperative for patients, their partners, and relatives, together with those responsible for their admission, to be aware that during hospitalization, they will merely try to restore conditions to allow the return to outpatient follow-up.

\section{Reasons for admission}

Any emergent situation that threatens the safety of the psychotherapeutic process and prevents continuing working productively is a reason for admission, for example, patients with chronic suicidality with the recent addition of neurovegetative symptoms and quasi-delirious concerns congruent with their mood. Uncertainty regarding the type, frequency, and degree of a pattern of abuse and dependence on alcohol or various substances; any complication that puts at risk the physical or mental integrity of the patient; diagnostic and interdisciplinary reassessment of complex mental situations with different comorbidities that could require close and long observation, as well as multiple consultations and highly specialized studies and instrumentation of complicated pharmacological regimens that merit around the clock follow-up, particularly in the context of varied medical comorbidities.

Patients with a history of multiple outpatient treatments, polypharmacy, and polydiagnosis, with very dysfunctional couples and/or families or that have never been informed of the underlying nature of a severe personality disorder and for whom, a comprehensive in-hospital evaluation is needed could be the starting 
point toward a psychoeducational shake-up that informs, reorients, and defines multiple diagnostic impressions and a prognosis that establishes the terms and conditions of treatment, and also the locus of responsibilities for all1,2,5,6.

The most frequent error in the hospitalization of patients with a BPO is that of not explaining what the diagnosis of a severe personality disorder means and involves. In contrast to the management of complications that forced the hospitalization or psychiatric comorbidities such as anxiety disorders, or "depression," often confused with a sense of emptiness, tedium, and boredom, characteristic of patients with a BPO and an identity diffusion syndrome ${ }^{2,5}$, diagnostic impressions should be clearly defined non-technically and with comprehensible terminology ${ }^{13}$. Follow-up strategies, the indications for available psychotherapeutic interventions with their respective limitations and scope, risks and benefits, and their potential side effects and adverse effects, or even contraindications, should also be explained.

The majority of patients with a BPO have a long history of being exploited with multiple treatments that were limited to complications and comorbidities. These create a quasi-parasitic passivity and conformity that never provide the opportunity for a coparticipative and comprehensive psychoeducational orientation that would allow them to know the underlying nature of their characterological disorder and, consequently, to aspire to a better quality of life and an independent level of functioning ${ }^{1,2,5,6,14}$.

\section{Suicidality}

Conventional hospitalization in a context of suicidality in a non-psychiatric medical ward should be limited to the treatment, repair, and restoration of bodily alterations secondary to the suicidal attempt. In this situation, the terms, conditions, and expectations of patients and staff are the same as those of a conventional admission. However, admitting a patient with a psychiatric disorder, and risk of suicidality, to a general hospital or a non-psychiatric ward for the prevention of suicide, is a mistake. Situations such as these require psychiatric hospitalization and specifically trained personnel.

The risk of suicidality appears with heterogeneous clinical manifestations: delirious psychotic and metabolic decompensation, neurocognitive dysfunction, brain tumors, affective disorders, and disabling major depressive disorder (MDD), among many others. Special attention should be given to carefully evaluate if the patient suffers from an alteration or deterioration of mental functioning that puts them at risk of losing volitional control. In this condition, the personnel assume the absolute responsibility of containing, protecting, and, if feasible, restoring the different coresponsible factors of this precarious functioning.

A challenging situation that requires thorough evaluation is the differential diagnosis of suicidality in the context of an incapacitating MDD and characterological suicidality (CS) without an MDD. In suicidality typical of a depressive condition related to a MDD, the following should be explored: the degree to which behavior and cognition has slowed down; if sadness has been replaced by a subjective experience of emptiness and a frozen mood, which borders on depersonalization and the loss of volitional control over one's person and life circumstances; the presence or absence of biological symptoms (reflected by sleep patterns and eating, weight, digestive functions, daily rhythmic variation of depressive affect, sexual desire, and muscle tone); and the overwhelming feeling that there is no possible alternative or no way out ${ }^{14}$.

Contrary to the prevailing belief that all ideation and suicidal behavior derive from "depressive" manifestations, the CS of patients with a BPO - not related to a comorbid MDD - is motivated by psychodynamics incited by anger, rage, hate, frustration, and envy ${ }^{14}$. Here, suicidal ideation could be at the service of controlling, punishing, torturing, and pleasure in taking revenge or demanding immediate help to manipulate or obtain the desired result.

Contrary to the traditional medical model, the psychiatric model differs for two reasons: (a) the medical model does not take into account that patients with a BPO intentionally place themselves at risk to provoke psychiatrists and staff to extend their time and emotional and therapeutic participation. Their excessive interventionism only leads to an increase of secondary gain as well as to a future self-perpetuating escalation of pathological acting out, affective storms, and suicidal threats ${ }^{2,5,6,14,15}$ and (b) the medical model recreates and perpetuates dependence and chronic emotional parasitism in BPO. The contemporary psychiatric model for $\mathrm{CS}$, on the other hand, promotes autonomy and reflective thinking ${ }^{1,14}$.

\section{CS elements of clinical judgment}

Even when CS occurs in very diverse clinical situations, with different meanings, and with different comorbidities, its understanding and management must 
consider the following common elements of clinical judgment: in CS, the patient has not lost volitional control of their life circumstances. He or she decides to end life or not. In the same way, he/she could decide voluntarily, at the moment, the specific place, and interpersonal context to carry it out. It is imperative not to forget that CS always involves a fantasized act of aggression not only toward oneself but also toward someone else or others, even if the patient denies it. If circumstances permit, in general, it is possible to recreate later the aggressively instigated scenarios of the desire to control, torture, reprimand, and harm others, and themselves, simultaneously. It is helpful to conceptualize CS as an intrapsychic and interpersonal problem with a role of aggressor and victim, but which could often oscillate capriciously. Therefore, a mandatory question when exploring these situations is: who in the internal world of the patient, or their external reality, is the potential target of their aggression, hatred, and/or envy ${ }^{2,14}$ ?

It must be investigated if CS tends to be chronic and even a way of living; a way to control, assure, and achieve everything that is desired. If the patient's CS overtime has become a sadistic instrument of control from which a huge secondary gain derives. A chronic pattern of CS at the service of manipulating and continuously maintaining omnipotent control over others must be differentiated from real emergencies, connected to other contingencies and human vicissitudes outside of its sadistic and volitional control.

Contrary to the severe decompensation of patients with bipolar disorder with loss of their volitional control, all borderline psychopathologies associated with CS are actualized in interpersonal settings, voluntarily selected by the patient, according to the nature of their own psychodynamic conflicts. In general, these interpersonal psychodynamics entail a clear and pleasant sadistic gratification of aggressive desires, as well as envy and contempt toward family members, couples, and all those in the area of mental health who are trying to help them.

Finally, although the psychodynamics of patients with BPO derive from split aspects of themselves (i.e., an aggressive part that wishes to annihilate and destroy and another that longs to live so that others will rescue and protect them), their internal conflict often culminates by projecting their ambiguous attitude in their external world; that is to say, in an external conflict between those patients who wish to destroy themselves and those "that prevent us from killing ourselves:" family, psychiatrists, psychotherapists, and hospital staff ${ }^{14}$.

\section{Therapeutic approach: strategic and tactical considerations}

Regardless of the administrative format and the model of hospital care - a conventional model of support, containment, and custody, a psychodynamically oriented therapeutic community (TC) or a mixed model of cognitive-behavioral techniques, mentalization, or a transference-focused psychotherapy extended to medical and psychiatric contexts - hospital and outpatient management of CS requires the following considerations:

Any manifestation of CS, even if apparently manipulative, must be taken seriously and explored. One in nine patients with BPO put an end to their existence. The real potential of lethality should be explored, as well as the degree of severity in previous suicidal attempts and the accessibility to the ways and means of doing so. If an intention of suicidality is present, what is its previous history: suicidality as a modus vivendi? What is the degree of patient control? Can he or she talk about their suicidal ideation, and can he or she guarantee that they feel under control. Do they admit that they feel out of control? In the latter, the possibility of immediate hospitalization should be proposed to a patient who asks for help and voluntarily accepts to sign a psychiatric hospitalization. If he or she refuses hospitalization, the patient and the family or couple will be informed that the responsibility of the clinicians and institution ends there. Often, patients with BPO who face the dilemma of being hospitalized, immediately say that it is "not that important and that they do not need hospitalization" that they feel "in control." Others sadistically hesitate and say "that they do not really know." With these, we must insist that if they are not sure of their volitional control, they should be hospitalized $^{14}$. If they refuse, it should be emphasized that at any time, they can come back and openly explore suicidal ideation.

In CS, every suicidal threat must be confronted openly. It is also imperative to explore and clarify the degree of volitional control and the locus of responsibility. Since CS is not related to a clear decompensation and loss of volitional control (e.g., due to a schizophrenic or a bipolar disorder, brain tumor, etc.), only he or she is responsible for requesting help inside or outside of the hospital. This approach must be repeated as many times as necessary. Frequently exposing patients with CS to the same strategy and tactics that systematically contribute to their assuming responsibility in controlling and containing their CS serves the following purposes: (a) it forces patients with CS to reduce their threats 
since it is anticipated that they will only be invited to confront their internal suicidal conflict with the same model of intervention; (b) the confrontation and delimitation of responsibilities reposition the locus of control and responsibility on patients. In the same way, it detracts from their sadistic control and the secondary gain derived from it ${ }^{5,6}$; and (c) the final result is a dissuasive impact that prevents patients and staff from being placed in the same anxiogenic and self-perpetuating vicious cycle, generated by their CS.

Concerning the locus of responsibility for CS, it is necessary to make explicit, to the patients, relatives, and their partners because it is volitional suicidality, only the patient is responsible for preserving their physical and mental integrity. Hospital staff cannot guarantee to predict or prevent suicide; only patients can ${ }^{2,14}$.

The most frequent and serious error in the hospital management of CS is not having clarified the objectives, terms, and conditions of hospitalization as well as the expectations of patients, relatives, and couples, on the one hand, and the hospital staff, on the other. In cases of frank CS, it must be carefully explained to the patient, relatives, and couples before hospital admission or as soon as possible, ideally in a meeting that includes the attending psychiatrist/resident, the head of nursing, and the hospital services coordinator that the purpose of hospitalization is not to guarantee that the patient will not commit suicide but to provide them with an institutional structure that supports and contains the problem, while continuing the exploration and treatment of the self-destructive nature of their character structure. A large number of families and couples and even some patients understand this institutional position. Others reject it, arguing that they will seek help in another place that guarantees the patient's life.

Of course, there will always be someone or another institution that will exploit the chronic affliction of families and couples, offering them pseudosecurity of preservation of patients with CS. In fact, when conflicts of interest appear, it is common to observe how some of these "saviors" become "fire extinguishers," who are always available for chronically distressed families, but at the expense of promoting an obligatory dependency and a self-perpetuating sadistic gratification in patients, derived from the exploitation of their suicidality as a torturing instrument of control and manipulation.

When the terms, conditions, expectations, and objectives of the hospitalization are not clarified, the risk exists of recreating the same chaotic situation that the patient left behind, but now in their relationship with other patients and the staff. If the locus of responsibility is not specified, it is impossible to not be the target of the sadistic control of patients with BPO and CS; therefore, it is not possible to have a minimum of security and serenity that allow the staff to reflect or implement strategies, tactics, and treatment techniques. When the objectives, terms, and conditions of admission are not explicit, patients, family, and staff take it as a guarantee that the institution assumes complete responsibility.

In the case of CS, it is necessary to be mentalized to lose a potentially suicidal patient ${ }^{14}$; even to be prepared to have to admit to a patient who presses to be told that we care about them if they commit suicide, that in fact, it will always be painful to lose a patient, but that we will continue with our lives, taking care of our patients, families, and ourselves. It is not possible to handle the sadistic and aggressive side that involves all CS if one is not mentalized for the loss of a patient with $\mathrm{CS}^{5,14}$.

Patients with phenotypic variants of the heterogeneous and protean spectrum of bipolar psychopathology, with a history of genuinely depressive suicidality but with an aggressively gratifying and sadistically instigated pattern of CS during periods between episodes of bipolar decompensation, represent a special challenge. In the suicidal behaviors of these patients, the classical tetrad of CS is observed: (a) the preservation of volitional control over their persona and life circumstances, which allows an orchestrated planning of the "grand finale;" (b) the cold and specifically instrumented selection of the moment, place, and interpersonal context that they want to affect in particular; (c) the egosyntonic expression of sadism, reflected in fantasies of inculpating, torturing, punishing, controlling or hurting those "aggressors" that in some way they think, victimized, mistreated, did not understand them, or failed them; and finally, (d) the consubstantial contradiction of all CS: although the suicidal behavior actualized a desire to detach oneself and destroy others of pain (the aggressively invested side of their intrapsychic conflict), the suicidal behavior also expresses and communicates the desire that those who survive carry them in their mind for the rest of their days (the primitive and idealized side of the conflict): "I leave you, but you will carry me in your minds for the rest of your lives"5. Contrary to the oversimplification that aggression in people is reactive or secondary to frustration ${ }^{16,17}$, the human brain shares with different mammal species, several neuroaffective systems in the periaqueductal gray matter of the midbrain ${ }^{18}$. Among others, one connected to the primary capacity to respond with fury, rage, and annihilatory violence before the potentially 
aversive. This primary neuroaffective system evolves toward the capacity to hate but is gradually modulated by the neuroregulatory, inhibitory functions of the cerebral cortex, as well as by high level inhibitory and sublimatory characterological defenses. At high levels of character consolidation, a stable and greater affective-cognitive regulation is observed. However, at low levels of personality organization, there is a greater affective-cognitive dysregulation and instability of personality functioning ${ }^{5,6}$.

Most people, including psychiatrists, therapists, and hospital staff, find it very egodystonic to speak, explore. and confront the unregulated, and even egosyntonic, expressions of rage, anger, and intense hate in patients with BPO and CS. However, patients with CS clearly sense the fear, in therapists and staff, of openly talking about their propensity to commit suicide and the manifestations of hatred, envy, and contempt that this entails. This only encourages them to further escalate their level of violence. For this reason, patients and staff in the TC often end up reproducing the same silent fear of couples and families outside of the hospital. They live with the exhausting anguish of being on the verge of losing a patient but are literally paralyzed to confront and reflect about it. In contrast, a timely confrontation, at all levels, by the staff within the TC, of a suicide threat, repositioning as soon as possible the limits and locus of control and responsibility in patients and families, slows down the gratification of the omnipotent and sadistic control of the entire TC by patients with $\mathrm{CS}^{5,14}$.

Because in patients with BPO, the deliberately sadistic expression of anger and violence, verbal and physical, toward others, is ostensibly egosyntonic, "justified" and volitional, the direct accessibility in patients with a BPO to explore its content with relative ease should not be forgotten. This receptiveness and freedom to do so contrast, however, with the defensive reticence of psychotherapists, psychiatrists, and staff, which impede them from exploring it and talk about it. Even in the grand rounds sessions of the hospital, where complex mental situations are presented, in which two or three comorbidities with a severe character pathology and CS coexist, the tendency is to focus on the narrative and discussion around one of the peripheral diagnoses panic attacks, post-traumatic stress syndrome, complex trauma, and treatment-resistant depression - but at the cost of ignoring the centrality of CS as a pervasively dominant symptom of a severe personality disorder.

The affective and ideational content underlying the psychodynamics connected to CS is accessible to exploration, both with mentalization techniques (MT) and with tactical and technical principles of transference-focused psychotherapy extended. These are applicable in psychiatric and medical contexts, outside of conventional psychotherapeutic processes, as in the TC and psychiatric wards ${ }^{1}$. In fact, of the first two segments of the interpretative process, in the latter, clarifying and confronting (before the interpretive activity proper, which comes later), overlap with $\mathrm{MT}^{1}$. However, it is the culmination of the whole integrative process in the exploratory activity and working through of split-off and conflictive aspects (segments three and four of the entire interpretative process), which finally encourages the increase in reflective thinking that might promote continuous emotional growth $2,14,19$.

It is evident that the treatment of CS should begin with the planning of the admission itself. However, the pathological collusion among all to avoid confronting this not only spoils an opportune moment of therapeutic potential but also opens the door to the toxic absorption of CS in the hospital environment. Repositioning the locus of control and the elucidation of responsibilities as well as the terms and conditions from the moment of admission allows aligning the interests of all toward a truly therapeutic approach to CS. In summary, the open confrontation of SC during the process of admission seeks the following objectives: (a) to increase collective awareness that SC is a volitional act and that only the patient can guarantee their physical and mental integrity; (b) to subtract power from the use of suicidal threats as a sadistic instrument of omnipotent control and enhance their reflective thinking; (c) to reduce the guilt in couples and relatives, and confront them with an inescapable reality: no one can guarantee absolute certainty of being able to avoid a situation that only the patient controls and is in a position to prevent; and finally, (d) to avoid the persecutory anxieties of the staff, generated by the fear of losing a patient, allowing them to preserve the minimum security and serenity essential for their therapeutic tasks.

\section{Final considerations}

Despite significant advances in organizational and administrative theory of psychiatric institutions, the psychology of small and large groups, systems theory, and its capitalization in the therapeutic use of social subsystems $\mathrm{s}^{6,8,20-22}$, as well as a true breakthrough in the consolidation of psychodynamic models of psychotherapeutic interventions derived from empirical evidence and systematically manualized for its generalized application, both in in-hospital treatment programs and in 
teaching and research ${ }^{1,23,24}$, the enormous gap between these advances and their more universal application to the contemporary psychiatric hospital is disconcerting.

Consequently, the non-delimited and confusing transposition of traditional medical models and more contemporary psychiatric approaches prevail: pharmacological and custodial control of patients; the unassimilated salads of multiple psychotherapeutic modalities without an integrating and holistic framework that systemizes them; and the wild mix of techniques and tactics that nullify each other and that result iatrogenic for both patients and staff.

Contrary to the prediction of few decades ago regarding the disappearance of the psychiatric hospital, the need for modern psychiatric services that integrate the advancement of contemporary proposals in the area of mental health and behavioral sciences is more imperative than ever. A continuous effort is required to critically explore the current situation and address the challenges represented by training personnel, professors, and residents in psychiatry for the optimal use of available hospital resources.

\section{Acknowledgments}

My deepest sense of gratitude to Sergio Lozano-Rodríguez, M.D. for his patience and expertise in the translation and editing of this manuscript.

\section{Conflicts of interest}

The author declares that there are no conflicts of interest.

\section{Funding}

No funding was received for this work.

\section{Ethical disclosures}

Protection of human and animal subjects. The authors declare that the procedures followed were in accordance with the regulations of the relevant clinical research ethics committee and with those of the Code of Ethics of the World Medical Association (Declaration of Helsinki).
Confidentiality of data. The authors declare that no patient data appear in this article.

Right to privacy and informed consent. The authors declare that no patient data appear in this article.

\section{References}

1. Hersh RG, Caligor E, Yeomans FE. Fundamentals of Transference-focused Psychotherapy: applications in Psychiatric and Medical Settings. New York: Springer; 2017.

2. Caligor E, Kernberg OF, Clarkin JF, Yeomans FE. Psychodynamic Therapy for Personality Pathology: treating Self and Interpersonal Functioning. Washington, D.C.: American Psychiatric Publications; 2018.

3. Paris J. Is hospitalization useful for suicidal patients with borderline personality disorder? J Pers Disord. 2004;18:240-7.

4. Fowler JC, Clapp JD, Madan A, Allen JG, Frueh BC, Fonagy P, et al A naturalistic longitudinal study of extended inpatient treatment for adults with borderline personality disorder: an examination of treatment response, remission and deterioration. J Affect Disord. 2018;235:323-31.

5. Guerrero CG. Trastornos de la Personalidad: avances y Desafíos Contemporáneos (en Psiquiatría, Psicología y Psicoanálisis). Mexico City: Editores de Textos Mexicanos; 2017. p. 365.

6. Garza-Guerrero C. Desregulacion afecto-cognoscitiva en organización limítrofe de la personalidade y sobremedicacion: iatrogenia, ignorância e pseudoprofisionalismo. Med Univ. 2012;14:99-112.

7. Paris J. Treatment of Borderline Personality Disorder: a Guide to Evidence-based Practice. New York: Guilford Press; 2008.

8. Garza Guerrero C. El Superyó en la Teoría y en la Práctica Psicoanalíticas. Mexico City: Editorial Paidós; 1989.

9. Bateman A, Peter, Fonagy P. Psychotherapy for Borderline Personality Disorder. Oxford: Oxford University Press; 2004.

10. Linehan MM. Cognitive-behavioral Treatment of Borderline Personality Disorder. New York: Guilford; 1993.

11. Gunderson JG. Borderline Personality Disorder: a Clinical Guide. Washington, D.C.: American Psychiatric Publishing; 2009.

12. Gunderson JG. Handbook of Good Psychiatric Management for Borderline Personality Disorder. Washington, D.C.: American Psychiatric Publishing; 2014

13. Shevrin $H$, Shectman $F$. The diagnostic process in psychiatric evaluations. Bull Menninger Clin. 1973;37:451-92.

14. Yeomans FE, Clarkin JF, Kernberg OF. Transference-focused Psychotherapy for Borderline Personality Disorder: a Clinical Guide. Washington, D.C.: American Psychiatric Publishing; 2015.

15. Clarkin JF, Yeomans FE, Kernberg OF. Psychotherapy for Borderline Personality: focusing on Object Relations. Washington, D.C.: American Psychiatric Publishing; 2006.

16. Masterson JF, Rinsley DB. The borderline syndrome: the role of the mother in the genesis and psychic structure of the borderline personality. Int J Psychoanal. 1975;56:163-77.

17. Buie $\mathrm{DH}$, Adler $\mathrm{G}$. Definitive treatment of the borderline personality. Int J Psychoanal Psychother. 1982;9:51-87.

18. Panksepp J, Biven L. The Archaeology of Mind: neuroevolutionary Origins of Human Emotions. New York: WW Norton and Company; 2012

19. Caligor E, Diamond D, Yeomans FE, Kernberg OF. The interpretive process in the psychoanalytic psychotherapy of borderline personality pathology. J Am Psychoanal Assoc. 2009:57:271-301.

20. Kernberg OF. Treatment of Severe Personality Disorders: resolution of Aggression and Recovery of Eroticism: American Psychiatric Association Publishing; 2018.

21. Garza-Guerrero AC. Theraputic use of social subsystems in a hospital setting. J Natl Assoc Priv Psychiatr Hosp. 1975;7:23-30.

22. Kernberg OF. Severe Personality Disorders: psychotherapeutic Strategies. New Haven: Yale University Press; 1986.

23. Zerbo E, Cohen S, Bielska W, Caligor E. Transference-focused psychotherapy in the general psychiatry residency: a useful and applicable model for residents in acute clinical settings. Psychodyn Psychiatry. 2013;41:163-81.

24. Bernstein J, Zimmerman M, Auchincloss EL. Transference-focused psychotherapy training during residency: an aide to learning psychodynamic psychotherapy. Psychodyn Psychiatry. 2015;43:201-21. 